NBER WORKING PAPER SERIES

\title{
UNDERSTANDING THE NEW-KEYNESIAN MODEL WHEN MONETARY POLICY SWITCHES REGIMES
}

\author{
Roger E.A. Farmer \\ Daniel F. Waggoner \\ Tao Zha \\ Working Paper 12965 \\ http://www.nber.org/papers/w12965
}

\author{
NATIONAL BUREAU OF ECONOMIC RESEARCH \\ 1050 Massachusetts Avenue \\ Cambridge, MA 02138 \\ March 2007
}

We thank Zheng Liu and Richard Rogerson for helpful discussions. The views expressed herein do not necessarily reflect those of the Federal Reserve Bank of Atlanta nor those of the Federal Reserve System. Farmer acknowledges the support of NSF grant SBR 0418174. The views expressed herein are those of the author(s) and do not necessarily reflect the views of the National Bureau of Economic Research.

(C) 2007 by Roger E.A. Farmer, Daniel F. Waggoner, and Tao Zha. All rights reserved. Short sections of text, not to exceed two paragraphs, may be quoted without explicit permission provided that full credit, including $\odot$ notice, is given to the source. 
Understanding the New-Keynesian Model when Monetary Policy Switches Regimes

Roger E.A. Farmer, Daniel F. Waggoner, and Tao Zha

NBER Working Paper No. 12965

March 2007

JEL No. E3,E5,E52

\section{ABSTRACT}

This paper studies a New-Keynesian model in which monetary policy may switch between regimes. We derive sufficient conditions for indeterminacy that are easy to implement and we show that the necessary and sufficient condition for determinacy, provided by Davig and Leeper, is necessary but not sufficient. More importantly, we use a two-regime model to show that indeterminacy in a passive regime may spill over to an active regime, no matter how active the latter regime is. As a result, a passive monetary policy is more damaging than has been previously thought. Our results imply that the propagation of shocks in an active regime, such as that of the Federal Reserve in the post-1982 period, may be substantially affected by the possibility of a return to a passive regime of the kind that was followed in the 1960s and 1970s.

Roger E.A. Farmer

UC, Los Angeles

Department of Economics

Box 951477

Los Angeles, CA 90095-1477

and NBER

rfarmer@econ.ucla.edu

Daniel F. Waggoner

1000 Peachtree Street N.E.

Atlanta

Georgia 30309-4470

dwaggoner@frbatlanta.org
Tao Zha

Federal Reserve Bank of Atlanta 1000 Peachtree Street N.E.

Atlanta, Georgia 30309-4470

tzha@earthlink.net 


\section{INTRODUCTION}

The basic new-Keynesian model (NK) consists of a forward-looking IS curve, an expectations-augmented Phillips curve and a policy rule, in which the interest rate responds to current values of inflation and output. This model is at the core of a wide class of dynamic stochastic general equilibrium (DSGE) models currently in use for policy analysis in both academia and central banks. ${ }^{1}$ A monetary policy rule that directs the policy-maker to respond to inflation by raising the interest rate less than one-for-one in response to an increase in inflation is said to be passive and a rule that directs the central bank to raise the interest rate more than one-for-one is said to be active (Leeper, 1991). A central bank that adopts an active rule is said to follow the Taylor principle after work by John Taylor (1993) who argued that a simple rule of this kind is a good characterization of actual central bank policy. In the basic NK model, passive policies lead to the existence of indeterminate equilibria in the sense that arbitrarily close to one equilibrium there is another one.

It is widely believed that the presence of indeterminacy is undesirable not only because it permits the existence of non-fundamental shocks but also because it amplifies the persistence and volatility of the equilibrium paths of inflation, interest rates, and output in response to fundamental shocks. ${ }^{2}$ Clarida, Galí, and Gertler (2000), Lubik and Schorfheide (2004), and Boivin and Giannoni (2006) estimate central bank policy rules for the U.S. economy for the period from 1960 through 1996. Their estimates show that macroeconomic volatility has been much lower in the post-1982 period than in the pre-1980 period and they attribute this reduction in volatility to the switch from a passive monetary policy, that implies indeterminacy, to an active policy that implements the unique equilibrium.

Motivated by this empirical work, Davig and Leeper (2005, 2006b, DL) extend the basic NK model by removing the assumption that a policy rule must be fixed forever. They study two policy regimes, one active in which policy is chosen by an inflation hawk and the other passive in which it is chosen by an inflation dove. DL allow the coefficients of the Taylor rule to vary stochastically across regimes according to a Markov-switching process and, within this Markov-switching NK model (MSNK), they assume that the inflation dove chooses a policy that would lead to indeterminacy if the economy were to remain forever in the passive regime and the inflation hawk chooses a policy that would lead to determinacy under a permanently active regime. ${ }^{3}$

\footnotetext{
${ }^{1}$ King (2000) and Woodford (2003) provide good introductions to the basic three-equation newKeynesian model.

${ }^{2}$ For a more detailed exposition of this argument see Woodford (2003, page 88).

${ }^{3}$ Economic arguments for modelling policy changes in a probabilistic manner were first put forth by Sims (1982) and by Cooley, LeRoy, and Raymon (1984). These authors argued that once a policy regime has changed, the rational public will expect such shifts to occur again in the future and will
} 
DL provide a necessary and sufficient condition for uniqueness of equilibrium of the MSNK model and they show that, for this model, the parameter region of determinate equilibria may be considerably larger than the union of the determinacy regions of the two separate NK models where agents do not take account of the probability of future regime change. In the MSNK model, if the passive regime is relatively shortlived and/or if the inflation hawk follows a sufficiently active policy, expectations about future regime change may induce the passive regime to become determinate. This effect, referred to by DL as a cross-regime spillover, occurs because rational agents take account of the probability of future regime change when forming their expectations.

Throughout the paper we maintain the same assumptions as DL. Specifically, we assume Ricardian fiscal policy and consider only bounded equilibria. Even with these qualifications, the MSNK model is different from the basic NK model in important ways since its determinacy and indeterminacy properties depend not only on parameters that describe how policy makers act in any given regime, but also on parameters that describe the probabilities of regime change. We would like to explain the equilibrium characteristics in terms of these underlying parameters and, in this paper, we make four significant contributions to this goal.

First, we derive a wide class of sufficient conditions for indeterminacy of the MSNK model that are straightforward to check in practice and that could potentially be used to assist a central banker to form policies that eliminate expectations-driven equilibria. Second, we prove that Davig and Leeper's condition, when used for identifying indeterminacy, is a special case of our general sufficient conditions. Moreover, we show that their condition is necessary but not sufficient to ensure local uniqueness. ${ }^{4}$ Third, we provide a calibrated example of an indeterminate equilibrium in which passive monetary policy spills over into the active regime and both regimes are indeterminate. ${ }^{5}$ This example is based on Lubik and Schorfheide's (2004) estimates and it is disturbing since it suggests that the U.S. economy may be in, or close to, the indeterminacy regime in practice. Finally, we show that if the probability of staying in the passive regime is sufficiently high or if policy in this regime is sufficiently passive, there may be nothing the inflation hawk can do to prevent indeterminacy even in the active regime. This new result implies that the mere existence of a sufficiently passive regime makes the effects of an inflation dove much more damaging than has been previously thought.

form a probability distribution over possible regime change. More recently, Leeper and Zha (2003) have drawn out implications of this way of thinking for practical monetary policy.

${ }^{4}$ See Galí (2006) for a similar point.

${ }^{5}$ Previous work by Farmer, Waggoner, and Zha (2006a) gives an example where indeterminacy, if it exists, occurs only in the passive regime. 
The rest of our paper is organized as follows. In Section II we present the newKeynesian model with regime switching. Section III discusses a claim of Davig and Leeper who provide a necessary and sufficient condition for the equilibrium of a model of this type to be unique. We use a calibrated example of the model to illustrate that their condition produces puzzling results. These results suggest the DL condition is not sufficient to rule out (local) nonuniqueness and in Section IV we substantiate this conjecture by providing a wider class of sufficient conditions for indeterminacy that applies to a broader class of Markov switching DSGE models of which the NK model is a member. In Section $\mathrm{V}$ we apply our theorem to a calibrated model of the U.S. economy using parameter values taken from the empirical work of Lubik and Schorfheide (2004) and we show that the equilibrium of the calibrated model is indeterminate and hence there may be sunspot equilibria in both the passive and the active regime. Section VI provides more examples in which once again there is a sunspot equilibrium in both the active and in the passive regime. Most of the examples illustrate a powerful implication from one of the corollaries of our main theorem. They differ from the example of Section $\mathrm{V}$ by demonstrating that if the inflation dove is sufficiently passive, there may be no feasible action that can be taken by the inflation hawk to restore determinacy of equilibrium. Section VII summarizes our results and makes some suggestions for extensions.

\section{THe MODEL}

We consider the new-Keynesian DSGE model estimated by Lubik and Schorfheide (2004) and analyzed by Davig and Leeper. This model is described by following equations,

$$
\begin{aligned}
\text { AS curve } & \pi_{t}=\beta E_{t} \pi_{t+1}+\kappa x_{t}+u_{t}^{S}, \\
\text { IS curve } & x_{t}=E_{t} x_{t+1}-\sigma^{-1}\left(i_{t}-E_{t} \pi_{t+1}\right)+u_{t}^{D}, \\
\text { Policy rule } & i_{t}=\alpha_{s_{t}} \pi_{t}+\gamma_{s_{t}} x_{t},
\end{aligned}
$$

where $x_{t}$ is output, $\pi_{t}$ is inflation, $i_{t}$ is the nominal interest rate, $u_{t}^{D}$ is an aggregate demand shock, and $u_{t}^{S}$ is an aggregate supply shock. Following DL, we measure the variables $\pi_{t}$ and $i_{t}$ as percentage deviations from their steady state values and $x_{t}$ as the deviation of output from its trend path.

The private sector block, consisting of Equations (1) and (2), has three regimeindependent parameters, $\sigma, \beta$ and $\kappa$. The parameter $\sigma$ represents the intertemporal elasticity of substitution, $\beta$ is the discount factor of the representative household, and $\kappa$ is the slope of the Phillips curve. Uncertain monetary policy is represented by Eq (3), the policy rule. This equation has two regime-dependent parameters $\left(\alpha_{s_{t}}\right.$ and $\gamma_{s_{t}}$ ) that capture the degree to which monetary policy is active or passive. We follow DL and assume that $s_{t}$ follows an exogenous Markov process with transition matrix 
$P=\left[p_{i j}\right]$. The element $p_{i j}$ represents the probability that $s_{t}=j$ given $s_{t-1}=i$ for $i, j \in\{1, \ldots h\}$ where $h$ is the number of regimes. For all examples considered in the paper, we focus on $h=2$ where monetary policy is active in the first regime and passive in the second regime.

To write the new-Keynesian model in a compact form, we substitute Eq (3) into Eq (2). Rearranging the terms in Eqs (1)-(2), the model can be written as

$$
F_{s t} y_{t}=H E_{t} y_{t+1}+u_{t}
$$

where

$$
\begin{aligned}
y_{t}=\left[\begin{array}{l}
\pi_{t} \\
x_{t}
\end{array}\right], & u_{t}=\left[\begin{array}{c}
u_{t}^{S} \\
u_{t}^{D}
\end{array}\right], \\
F_{s_{t}}=\left[\begin{array}{cc}
1 & -\kappa \\
\sigma^{-1} \alpha_{s_{t}} & 1+\sigma^{-1} \gamma_{s_{t}}
\end{array}\right], & H=\left[\begin{array}{cc}
\beta & 0 \\
\sigma^{-1} & 1
\end{array}\right] .
\end{aligned}
$$

The remainder of the paper is based on this MSNK model and our examples in Sections V and VI use the model to make a number of points. The sufficiency theorem of Section IV is more general and allows for $h$ regimes and $n$ equations in each regime.

\section{Some Puzzling Results}

A key policy question is: Under what condition is the equilibrium of the MSNK model (locally) non-unique in the sense that arbitrarily close to it there is another one? This question is at the heart of our subject because the design of a good policy depends on it. By avoiding policies that lead to locally non-unique equilibria, a policy maker may hope to reduce the variance and persistence of output and inflation. Davig and Leeper $(2005,2006 \mathrm{~b})$ claim to give an answer to our question by providing a necessary and sufficient condition for uniqueness (and hence also for nonuniqueness) of equilibrium in the MSNK model represented by Eq (4). They refer to this condition as the "Long-Run Taylor Principle". In the rest of this section we review the DL claim and demonstrate that their claim has puzzling implications. ${ }^{6}$

III.1. The DL necessary and sufficient condition. The DL approach begins by expressing the conditional expectations $E_{t} \pi_{t+1}$ and $E_{t} x_{t+1}$ in $\mathrm{Eq}(4)$ as follows ${ }^{7}$

$$
\begin{aligned}
& E_{t} \pi_{t+1}=p_{s_{t} 1} E_{t} \pi_{1 t+1}+p_{s_{t} 2} E_{t} \pi_{2 t+1}, \\
& E_{t} x_{t+1}=p_{s_{t} 1} E_{t} x_{1 t+1}+p_{s_{t} 2} E_{t} x_{2 t+1},
\end{aligned}
$$

\footnotetext{
${ }^{6}$ DL's paper is part of a growing literature on Markov-switching rational expectations models that includes papers by Andolfatto and Gomme (2003), Leeper and Zha (2003), Schorfheide (2005), Svensson and Williams (2005), Farmer, Waggoner, and Zha (2006b), and Davig and Leeper (2006a).

${ }^{7}$ For complete details, see Appendix B in Davig and Leeper (2005, 2006b). For an analysis of why these expressions are problematic, see Appendix A at the end of our paper.
} 
where $\pi_{1 t}, \pi_{2 t}, x_{1 t}$, and $x_{2 t}$ are newly introduced random variables such that $\pi_{i t}=\pi_{t}$ and $x_{i t}=\pi_{t}$ when $s_{t}=i$ for $i=1,2$. Up to this point $\pi_{1 t}$ and $x_{1 t}$ have not been defined when $s_{t}=2$ and $\pi_{2 t}$ and $x_{2 t}$ have not been defined when $s_{t}=1$. Nonetheless, DL substitute Eqs (5) and (6) into the model, Eq (4), and they express each of the original two equations as a pair of equations in the four endogenous random variables $\pi_{1 t}, \pi_{2 t}, x_{1 t}$, and $x_{2 t}$. This procedure leads to the following expanded linear system:

$$
\underbrace{\left[\begin{array}{cccc}
1 & 0 & -\kappa & 0 \\
0 & 1 & 0 & -\kappa \\
\sigma^{-1} \alpha_{1} & 0 & 1+\sigma^{-1} \gamma_{1} & 0 \\
0 & \sigma^{-1} \alpha_{2} & 0 & 1+\sigma^{-1} \gamma_{2}
\end{array}\right]}_{B} \underbrace{\left[\begin{array}{c}
\pi_{1 t} \\
\pi_{2 t} \\
x_{1 t} \\
x_{2 t}
\end{array}\right]=}_{A} \underbrace{\left[\begin{array}{l}
E_{t} \pi_{1 t+1} \\
E_{t} \pi_{2 t+1} \\
E_{t} x_{1 t+1} \\
E_{t} x_{2 t+1}
\end{array}\right]+\left[\begin{array}{c}
u_{t}^{S} \\
u_{t}^{S} \\
u_{t}^{D} \\
u_{t}^{D}
\end{array}\right] .}_{\left.\begin{array}{cccc}
\beta p_{11} & \beta p_{12} & 0 & 0 \\
\beta p_{21} & \beta p_{22} & 0 & 0 \\
\sigma^{-1} p_{11} & \sigma^{-1} p_{12} & p_{11} & p_{12} \\
\sigma^{-1} p_{21} & \sigma^{-1} p_{22} & p_{21} & p_{22}
\end{array}\right]}
$$

Computing a uniqueness condition for this linear rational expectations system is a standard problem (see, for example, Sims (2002); Lubik and Schorfheide (2003)) and DL correctly state that a necessary and sufficient condition for determinacy of the equilibrium in $\mathrm{Eq}(7)$ is that all the generalized eigenvalues of $(B, A)$ lie inside the unit circle. However, this condition is not the same as finding a unique bounded equilibrium to the original model represented by Eq (4). ${ }^{8}$ Using the linear system, Eq (7), DL make the following claim.

Claim 1. A necessary and sufficient condition for the MSNK model, Eq (4), to have a unique bounded equilibrium is that all the generalized eigenvalues of $(B, A)$ lie inside the unit circle.

Using Claim 1, DL provide various determinacy regions for the MSNK model and reinterpret Lubik and Schorfheide (2004)'s empirical work in light of these regions. In Section VI, we will discuss some of their results in more detail.

III.2. An implication of the DL condition. In this subsection we provide an example of the NK model to illustrate a puzzling implication of the DL claim. Our example suggests the conjecture that their condition is not sufficient to ensure uniqueness of the equilibrium, a conjecture that we prove in Section IV.

\footnotetext{
${ }^{8}$ In the special case of the Fisherian model, for which $n=1$, the DL condition can be supplemented by the restriction that the scalar quantities $F_{s_{t}}^{-1} H$ are non-negative to provide a correct set of necessary and sufficient conditions for local uniqueness. This does not imply, even for this special case, that Eqs (7) and (4) equivalent.
} 
Consider the NK model with the parameter values reported in Table 1. All of these numbers are within the parameter space considered in the existing literature. They imply that the first regime is active and has a unique equilibrium when treated in isolation and the second regime is passive and indeterminate.

\begin{tabular}{|c|c|c|c|c|c|c|c|}
\hline \multicolumn{2}{|c|}{ Private Sector } & \multicolumn{2}{|c|}{ Regime 1 } & \multicolumn{2}{c|}{ Regime 2 } & Trans Prob \\
\hline \hline$\beta$ & $\sigma$ & $\kappa$ & $\alpha_{1}$ & $\gamma_{1}$ & $\alpha_{2}$ & $\gamma_{2}$ & $p_{22}$ \\
\hline 0.99 & 2.84 & 0.3 & 3.6 & 0.3 & 0.7 & 0.1 & 0.9 \\
\hline
\end{tabular}

TABle 1. Parameter Values for a NK Economy

For these parameter values, monetary policy reacts strongly to inflation in the active regime $\left(\alpha_{1}=3.6\right)$. Since the passive regime is persistent $\left(p_{22}=0.9\right)$, it seems likely that if the active regime is short-lived, the model will be indeterminate. This conjecture is supported by applying DL's necessary and sufficient condition which indicates that indeterminacy occurs if $0<p_{11} \leq 0.57$ since for these values of $p_{11}$, at least one of the generalized eigenvalues of $(B, A)$ lies outside the unit circle.

The logic of the DL argument implies that if the active regime is persistent enough, cross-regime spillovers may cause the equilibrium to be unique. The economic intuition for this switch to determinacy is that agents in the passive regime take into account the probability that monetary policy will become active. As DL argue persuasively, the more persistent is the active regime, the more likely will be the occurrence of cross-regime spillovers working through expectations-formation effects.

\begin{tabular}{|c|c|c|c|}
\hline Equilibrium characteristics & indeterminacy & determinacy & indeterminacy \\
\hline$p_{11}$ (Prob of active regime) & $(0,0.57]$ & {$[0.58,0.86]$} & {$[0.87,1.0]$} \\
\hline
\end{tabular}

TABLE 2. Misleading spillovers implied by the DL condition. As the active regime becomes progressively more persistent, the model moves from being indeterminate to determinate but then back into the region of indeterminacy.

Indeed, as the persistence of the active regime increases from the range $0<p_{11} \leq$ 0.57 to the range $0.58 \leq p_{11} \leq 0.86$, the DL condition implies that cross-regime spillovers take effect and the equilibrium moves from being indeterminate to determinate just as the logic of their argument suggests. Continuing to apply the same condition, however, one concludes that as the active regime becomes even more persistent with $p_{11}$ above 0.86 , the equilibrium reverts to indeterminacy. Table 2 summarizes these puzzling findings. 


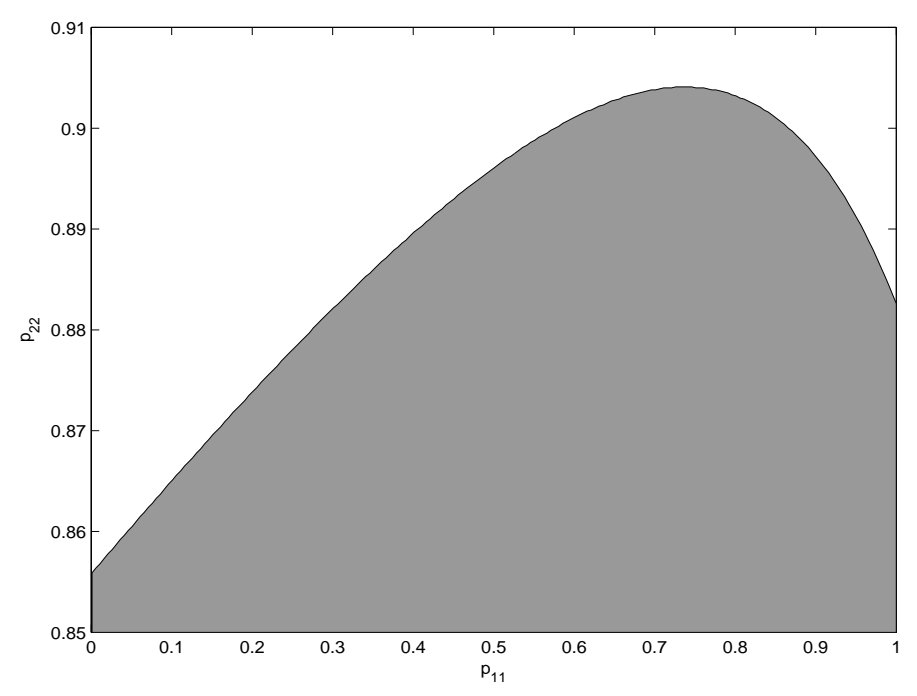

Figure 1. A problematic implication of the DL condition: the newKeynesian model with $\alpha_{1}=3.6, \alpha_{2}=0.7, \gamma_{1}=0.3, \gamma_{2}=0.1, \beta=$ $0.99, \sigma=2.84$, and $\kappa=0.3$. The shaded area is a "uniqueness equilibrium" region implied by the DL's necessary and sufficient condition for determinacy. As the active regime becomes progressively more persistent, the model moves from being indeterminate to determinate for some values of $p_{22}$. For other values of $p_{22}$, the model moves from the indeterminate region to the determinate region but then back into the region of indeterminacy.

Figure 1 plots the "determinacy" region implied by the DL condition for a range of values of $p_{11}$ and $p_{22}$. Clearly, some of the results implied by this figure are spurious since it makes no sense for the model to move from indeterminacy to determinacy and back to indeterminacy as the active regime increases in persistence. How does one explain this puzzle? We show in Section IV that the DL condition is a necessary but not a sufficient condition for the NK model to be determinate.

\section{Sufficient Conditions for Indeterminacy}

In this section we derive a sufficient condition for indeterminacy for a class of Markov-switching rational expectations models that includes the MSNK model as a special case. We show that our condition is equivalent to finding a solution to a certain non-linear equation and we derive two useful corollaries to our main theorem. The first shows that the DL condition is necessary for determinacy. The second provides a separate sufficient condition for indeterminacy that can be checked in practice. Using Corollary 2, one can easily construct counterexamples showing that the DL condition is not sufficient for determinacy. Proofs are collected in Appendices B-C. 
Consider models of the form

$$
\Gamma_{s t} y_{t}=E_{t} y_{t+1}+\Psi_{s_{t}} u_{t}
$$

where $y_{t}$ is an $n$-dimensional vector of endogenous random variables and $u_{t}$ is an $m$-dimensional vector of exogenous shocks which are allowed to be serially correlated. In particular, $u_{t}$ may take the vector autoregressive form $u_{t}=\rho u_{t-1}+\varepsilon_{t}$ where all the eigenvalues of $\rho$ are strictly less than one in absolute value and $\varepsilon_{t}$ is a bounded exogenous process independent of $s_{t}$ satisfying $E_{t-1}\left[\varepsilon_{t}\right]=0$. The new-Keynesian model, Eq (4), is a special case of Eq (8) where $\Gamma_{s_{t}}=H^{-1} F_{s_{t}}$ and $\Psi_{s_{t}}=H^{-1}$.

Let the notation $\operatorname{diag}\left(X_{i}\right)$ denote a block-diagonal matrix whose diagonal elements are $X_{1}, \ldots, X_{h}$. We have the following key theorem.

Theorem 1 . If there exist complex numbers $c_{1}, \cdots, c_{h}$ with $\left|c_{i}\right| \leq 1$ for all $i=1, \ldots, h$ and an $n h$-dimensional non-zero complex vector $v$ such that

$$
\left(\operatorname{diag}\left(\Gamma_{i}\right)-\left(\operatorname{diag}\left(c_{i}\right) P\right) \otimes I_{n}\right) v=0,
$$

then there exists multiple bounded solutions to Eq (8).

It is computationally feasible to check whether $\mathrm{Eq}(9)$ is satisfied by finding $c_{i}$ 's that solve the non-linear equation

$$
\operatorname{det}\left(\operatorname{diag}\left(\Gamma_{i}\right)-\left(\operatorname{diag}\left(c_{i}\right) P\right) \otimes I_{n}\right)=0
$$

subject to the constraint that $\left|c_{i}\right| \leq 1$ for all $i=1, \ldots h$. In the case of one regime this reduces to an eigenvalue problem. In the case of two or more regimes it is a polynomial that can be solved numerically, for example, by a grid search over the $h$ complex numbers $\left\{c_{i}\right\}_{i=1}^{h}$. In the two-regime NK model, if there exists a solution where both of the $c_{i}$ are inside the unit circle then our theorem guarantees the existence of multiple bounded sunspot solutions to the MSNK model in both the active and passive regimes. ${ }^{9}$

The following corollaries highlight two special cases of interest. The first shows that the DL condition is a necessary condition for uniqueness and the second can easily be used to construct examples to show that it is not sufficient.

Corollary 1. If $\operatorname{diag}\left(\Gamma_{i}^{-1}\right)\left(P \otimes I_{n}\right)$ has an eigenvalue greater than or equal to one in absolute value, then there are multiple bounded solutions to Eq (8).

It might not be immediately obvious that the condition in this corollary is the same as the DL condition when their condition is used to identify indeterminacy. But if

\footnotetext{
${ }^{9}$ One can show that for the case $n=1$, Theorem 1 is both necessary and sufficient for the existence of multiple bounded solutions. If one restricts attention to non-negative values for the scalars, $\Gamma_{i}$, then DL's condition for the Fisherian (one dimensional) model becomes a special case of this Theorem.
} 
one appropriately reorders the variables and equations in their expanded system, Eq (7), one will see that all the generalized eigenvalues of $(B, A)$ lie on or outside the unit circle if and only if the condition in Corollary 1 is satisfied.

Corollary 2. If there exists $i \in\{1, \ldots, h\}$ such that $\Gamma_{i}$ has an eigenvalue less than or equal to $p_{i i}$ in absolute value, then there are multiple bounded solutions to (8).

Corollary 2 implies that there are conditions based only on the parameters of a single regime that imply indeterminacy in every regime. This result is new. In Section VI, we give a number of parameterized examples of the MSNK model, based on this corollary, in which we show that if the inflation dove is sufficiently passive there may be no action that can be taken by the inflation hawk that will restore determinacy even in the active regime.

\section{Indeterminacy in An ACtive Regime}

In this section we use Theorem 1 to construct indeterminate solutions for both active and passive regimes in the NK model. We then provide a quantitative example using calibrated parameter values based on the estimates of Lubik and Schorfheide (2004). This example illustrates one of the key messages of this paper. Multiple self-fulfilling paths may spill over to the active regime even if the passive regime is relatively short-lived. Moreover, the parameter values for which this phenomenon occurs are well within the confidence bounds for recent parameter estimates based on U.S. data.

It is widely believed that U.S. monetary policy in the post-1982 period has been active enough to ensure uniqueness of the equilibrium within the framework of the NK model. In the regime-switching environment studied by DL, however, there exists a probability that policy will revert to the passive regime. If the equilibrium is not unique, does indeterminacy occur only in the passive regime so that the equilibrium remains unique once an inflation hawk takes office? This question is important because a positive answer implies that as long as the inflation hawk is in office, the propagation of shocks will not be affected by indeterminacy in the passive regime. Let bygones be bygones, so to speak. ${ }^{10}$ But if the answer to the question is negative, a passive monetary policy is more damaging and prevalent than had previously been thought because there may be multiple self-fulfilling paths for inflation even when an inflation hawk is appointed to run the central bank.

V.1. Multiple bounded equilibria. To maintain analytical clarity, we assume that the vector $u_{t}$ (of demand and supply shocks) follows an i.i.d. process. This assumption does not alter conditions under which the equilibrium may or may not be unique.

\footnotetext{
${ }^{10}$ Farmer, Waggoner, and Zha (2006a) give an example of this sort in the context of a very simple model.
} 
The minimum-state-variable solution to the NK model (4) is given by the expression

$$
y_{t}=G_{s_{t}} u_{t}
$$

where

$$
G_{s_{t}}=\Gamma_{s_{t}}^{-1} \Psi_{s_{t}}=\frac{1}{\sigma+\gamma_{s_{t}}+\kappa \alpha_{s_{t}}}\left[\begin{array}{cc}
\sigma+\gamma_{s_{t}} & \kappa \sigma \\
-\alpha_{s_{t}} & \sigma
\end{array}\right] .
$$

This solution exhibits no dynamics. If the sufficiency condition of Theorem 1 is satisfied, the solution is not unique and there are other bounded equilibria in which both inflation and output, represented by $y_{t}$, are serially dependent. Lubik and Schorfheide (2004) give an analytical form for serially-dependent sunspot equilibria for the basic NK model with no regime switching. We show, below, how to find a similar representation of sunspot equilibria for the MSNK model. In these equilibria, inflation and output are serially dependent even when the fundamental shocks are i.i.d.

To construct an indeterminate solution in the Markov-switching case let $c_{1}, c_{2}$ and $v^{\prime}=\left[v_{1}^{\prime}, v_{2}^{\prime}\right]$ satisfy Eq (9) of Theorem 1 . For this example, the vectors $v_{1}$ and $v_{2}$ are two-dimensional and since Eq (9) is satisfied, there exist multiple bounded solutions to the NK model, Eq (4), given by the expression,

$$
y_{t}=G_{s_{t}} u_{t}+w_{t}
$$

where

$$
w_{t}=\left(\frac{c_{s_{t-1}}}{v_{s_{t-1}}^{\prime} v_{s_{t-1}}} v_{s_{t}} v_{s_{t-1}}^{\prime}\right) w_{t-1}+v_{s_{t}}\left(M u_{t}+\xi_{t}\right) .
$$

We have represented the solution as the sum of the fundamental solution (which is the component $G_{s_{t}} u_{t}$ ) and a sunspot solution represented by $w_{t}$. The sunspot solution is autocorrelated and is driven by a non-fundamental sunspot shock, which is the term $\xi_{t}$, and a fundamental sunspot shock, which is the component $M u_{t}$ where $M$ is an arbitrary $1 \times 2$ row matrix. The non-fundamental shock, $\xi_{t}$, is any one-dimensional bounded stochastic process with zero mean that is independent of both $u_{t}$ and $s_{t}{ }^{11}$ The terms $c_{s_{t}}$ and $v_{s_{t}}$ are analogous to eigenvalues and eigenvectors in a model with no regime switching. Since the $c_{s_{t}}$ are all inside the unit circle, the sequence $\left\{w_{t}\right\}$ remains bounded and the sunspot shocks die out asymptotically, just as in the one-regime case studied by Lubik and Schorfheide (2003, 2004).

It is straightforward to verify that $\mathrm{Eq}(12)$ is a solution. Since the stochastic processes of $u_{t}$ and $\xi_{t}$ are independent of $s_{t}$, it can be easily seen that

$$
E_{t} y_{t+1}=\left(\frac{c_{s_{t}}}{v_{s_{t}}^{\prime} v_{s_{t}}} E_{t} v_{s_{t+1}} v_{s_{t}}^{\prime}\right) w_{t}
$$

\footnotetext{
${ }^{11}$ If $v_{s_{t-1}}=0$, then the coefficient of $w_{t-1}$ is taken to be zero and if any of the $c_{i}$ are equal to one in absolute value, then $M$ and $\xi_{t}$ should be taken to be zero in order to guarantee boundedness. If any of the $c_{i}$ or $v_{i}$ are complex, then the solution given by Eq (12) will be complex, but either the real or the imaginary component of $w_{t}$ can be used to construct real solutions.
} 
where

$$
E_{t} v_{s_{t+1}}=p_{s_{t} 1} v_{1}+p_{s_{t} 2} v_{2}
$$

Since $G_{s_{t}}=\Gamma_{s_{t}}^{-1} \Psi_{s_{t}}$, one can see from Eqs (8) and (12) that

$$
E_{t} y_{t+1}=\Gamma_{s_{t}} y_{t}-\Psi_{s_{t}} u_{t}=\Gamma_{s_{t}} w_{t} .
$$

It follows from $\mathrm{Eq}(9)$ that (12) is a solution if and only if

$$
\left(\frac{c_{s_{t}}}{v_{s_{t}}^{\prime} v_{s_{t}}}\left(p_{s_{t} 1} v_{1}+p_{s_{t} 2} v_{2}\right) v_{s_{t}}^{\prime}-\Gamma_{s_{t}}\right) w_{t}=0
$$

As $w_{t}$ is proportional to $v_{s_{t}}$, Eq (14) needs to be verified only for $w_{t}=v_{s_{t}}$. Because $c_{i}$ and $v_{i}$ are chosen to satisfy Eq (9), Eq (14) will also hold. Therefore, we can directly verify $\mathrm{Eq}(14)$ for a specific example, such as the one presented in the next subsection.

V.2. A Quantitative Example. This section demonstrates the existence of sunspot solutions to the NK model when parameters are calibrated to the values estimated by Lubik and Schorfheide (2004). Although these authors did not explicitly account for the probabilities of regime change in their empirical work, taking account of these probabilities in the NK model is a natural extension of their work. Our point is to show that the possibility that the set of regimes may be indeterminate is not outside of the bounds of plausibility and for this purpose the estimated values of Lubik and Schorfheide (2004) seem a good place to start.

Lubik and Schorfheide estimate a constant-parameter version of the NK model for the two subsamples: 1960:I-1979:II and 1982:I-1997:IV. For our calibrated example we chose policy parameters in each regime equal to their estimates and we set privatesector parameters equal to the means of their estimates for individual regimes. These values are reported in columns 1 through 7 of Table 3. Notice that in contrast to Table 1, Regime 1 is passive, in line with historical precedence (McChesney-MartinBurns-Miller came before Volker-Greenspan-Bernanke).

\begin{tabular}{|c|c|c|c|c|c|c|c|c|}
\hline \multicolumn{2}{|c|}{ Private Sector } & \multicolumn{2}{c|}{ Regime 1 } & \multicolumn{2}{c|}{ Regime 2 } & \multicolumn{2}{c|}{ Trans. Pr. } \\
\hline \hline$\beta$ & $\sigma$ & $\kappa$ & $\alpha_{1}$ & $\gamma_{1}$ & $\alpha_{2}$ & $\gamma_{2}$ & $p_{11}$ & $p_{22}$ \\
\hline 0.9949 & 1.6550 & 0.6750 & 0.7700 & 0.1700 & 2.1900 & 0.3000 & 0.8577 & 0.9900 \\
\hline
\end{tabular}

TABle 3. Parameter Values Calibrated to Lubik and Schorfheide (2004)'s Estimates

Columns 8 and 9 of this table report our calibrated transition probabilities. Given that rational agents experienced passive monetary policy in the past, they may still fear, under the Volker-Greenspan-Bernanke regime, that a future policymaker may deviate from its active stance. Some deviations may simply reflect the reality that the policymaker has a mandate to address other economic concerns such as recessions or 
financial crises. Moreover, since Chairman of the Federal Reserve Board is a political appointee, there is always a probability that an inflation dove may be appointed to run the Fed. To take account of these possibilities we chose transition probabilities as $p_{11}=0.8577$ and $p_{22}=0.99$.

The choice of $p_{11}$ implies that agents perceive a $14 \%$ chance of returning to an active regime in any quarter. This choice implies an expected duration for the passive regime of only seven quarters. In contrast, the expected duration of the active regime $\left(p_{11}=\right.$ 0.99 ) is twenty five years. These numbers imply that, in the ergodic distribution, the economy will spend less than $7 \%$ of the time in the hands of the inflation dove. These numbers do not seem unreasonable given historical experience.

Taking our calibrated parameterization, we solve Eq (10) and we find numbers $\left|c_{1}\right|<1$ and $\left|c_{2}\right|<1$ such that

$$
\operatorname{det}\left(\operatorname{diag}\left(\Gamma_{i}\right)-\left(\operatorname{diag}\left(c_{i}\right) P\right) \otimes I_{n}\right)
$$

is equal to zero. Our algorithm leads to the following numerical solutions:

$$
\begin{gathered}
c_{1}=0.999795, \\
c_{2}=0.738137, \\
v_{1}=\left[\begin{array}{l}
-0.977509 \\
-0.210551
\end{array}\right], \\
v_{2}=\left[\begin{array}{l}
-0.010062 \\
0.0065658
\end{array}\right] .
\end{gathered}
$$

Substituting these values into Eq (13) and using Eq (12), we find persistent sunspot solutions that remain bounded in both active and passive regimes. ${ }^{12}$ This example highlights a key finding of our work. Even though a passive regime may be short-lived and the agents' belief in switching to this regime is low, the possibility that a passive policy may be adopted at times makes it difficult for an active policy to eliminate the destabilizing effects of self-fulfilling expectations.

\section{Further Implications of Cross-Regime Spillovers}

In this section, we discuss a number of examples of cross-regime spillovers in the MSNK model. The purpose is two-fold. One is to show practical problems of using Corollary 1 as a necessary and sufficient condition for identifying indeterminacy (and hence also for identifying determinacy), as was proposed in the existing literature (Davig and Leeper, 2005, 2006b). The other is to draw out a disturbing implication

\footnotetext{
${ }^{12}$ It is straightforward to verify that the resulting expression obtained by substituting these values into Eqs (12) and (13) satisfies Eq (8) as required.
} 
of Corollary 2. Consider the situation where there is a passive regime that satisfied Corollary 2 but the parameter values are such that Corollary 1 is violated. If one uses Corollary 1 as though it were a necessary and sufficient condition for indeterminacy, one may wrongly conclude that an inflation hawk can make the policy active enough to ensure uniqueness of the equilibrium. A correct conclusion is: there are no possible values for the parameters of the interest rate and output-gap response coefficients of the Taylor rule in the active regime that can restore determinacy.

We begin by revisiting the example considered in Section III.2 and we demonstrate that this example satisfies the conditions of Corollary 2. Recall that in this example, regime 1 is active and regime 2 is passive. Substituting the parameter values from Table 1 into $F_{2}$ and $H$ into Eq (4) and computing $\Gamma_{2}=H^{-1} F_{2}$, one can verify that $\Gamma_{2}$ has an eigenvalue less than $p_{22}=0.9$. By Corollary 2, the equilibria of the model are indeterminate regardless of the values of $p_{11}$ and bounded sunspot solutions can be computed from Eq (12). Table 4 summarizes the correct equilibrium properties of this model for different values of $p_{11}$. This result explains the puzzling results displayed in Table 2, which arise from using DL's incorrect sufficiency condition for uniqueness.

\begin{tabular}{|c|c|c|c|}
\hline Equilibrium characteristics & indeterminacy & indeterminacy & indeterminacy \\
\hline$p_{11}$ (Prob of active regime) & $(0,0.57]$ & {$[0.58,0.86]$} & {$[0.87,1.0]$} \\
\hline
\end{tabular}

TABlE 4. Correct equilibrium characteristics of the example in Section III.2 (according to Corollary 2).

When $0.58 \leq p_{11} \leq 0.86$, the model has multiple bounded equilibria in contrast to the incorrect results reported in Table 2 . Figure 2 plots $p_{11}$ against $p_{22}$ holding other parameter values fixed. The union of the dark-shaded and light-shaded areas indicates an indeterminacy region. The light-shaded area is determined by Corollary 1 of Theorem 1. The dark-shaded area, determined by Corollary 2, is incorrectly identified as a "uniqueness" region by the DL condition stated in Claim 1.

Figure 3 displays an indeterminate region corresponding to different values of $\alpha_{1}$ and $\alpha_{2}$ when $p_{11}=0.8$ and $p_{22}=0.9$. The values of the other parameters are the same as in Table 1. As long as $\alpha_{1}>1$, the model would be determinate in regime 1 if $s_{t}=1$ were an absorbing regime. Similarly, for $0<\alpha_{2}<1$ the model would be indeterminate if $s_{t}=2$ were absorbing. The union of dark-shaded and light-shaded areas (computed using Corollaries 1 and 2) identifies a region of indeterminacy for the MSNK model. The dark-shaded area represents the indeterminate region missed by using Corollary 1. In this region monetary policy is sufficiently passive $\left(\alpha_{2}\right.$ is less than 0.75 ), that there is no unique solution to the new-Keynesian model no matter how active is policy in the active regime. 


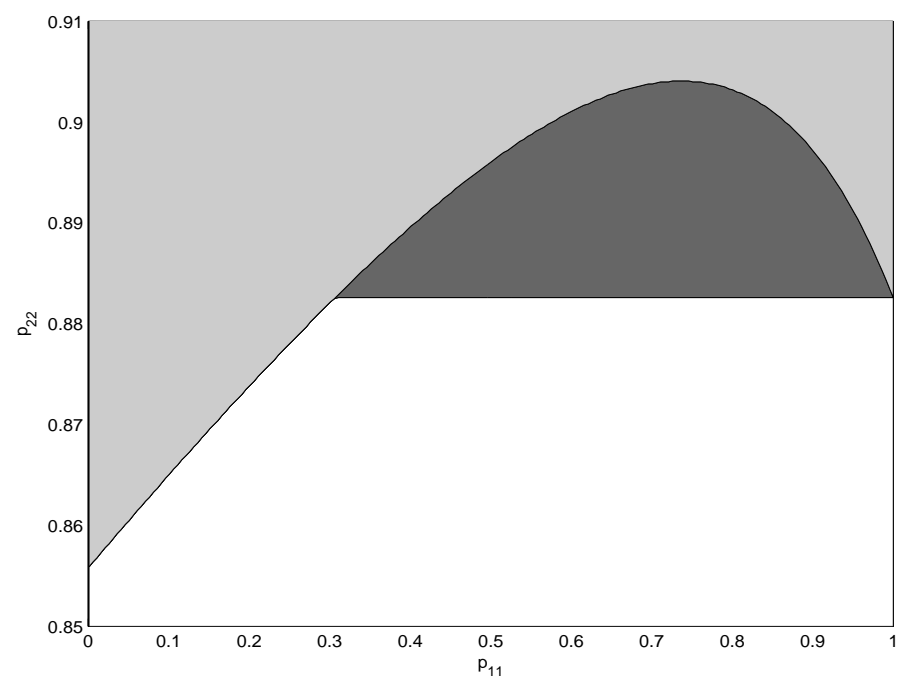

Figure 2. Equilibrium characteristics: the new-Keynesian model with $\alpha_{1}=3.6, \alpha_{2}=0.7, \gamma_{1}=0.3, \gamma_{2}=0.1, \beta=0.99, \sigma=2.84$, and $\kappa=0.3$. The union of the dark and light shaded areas is an indeterminacy region according to Corollaries 1 and 2. The dark-shaded area is incorrectly regarded as a "uniqueness" region by the DL condition.

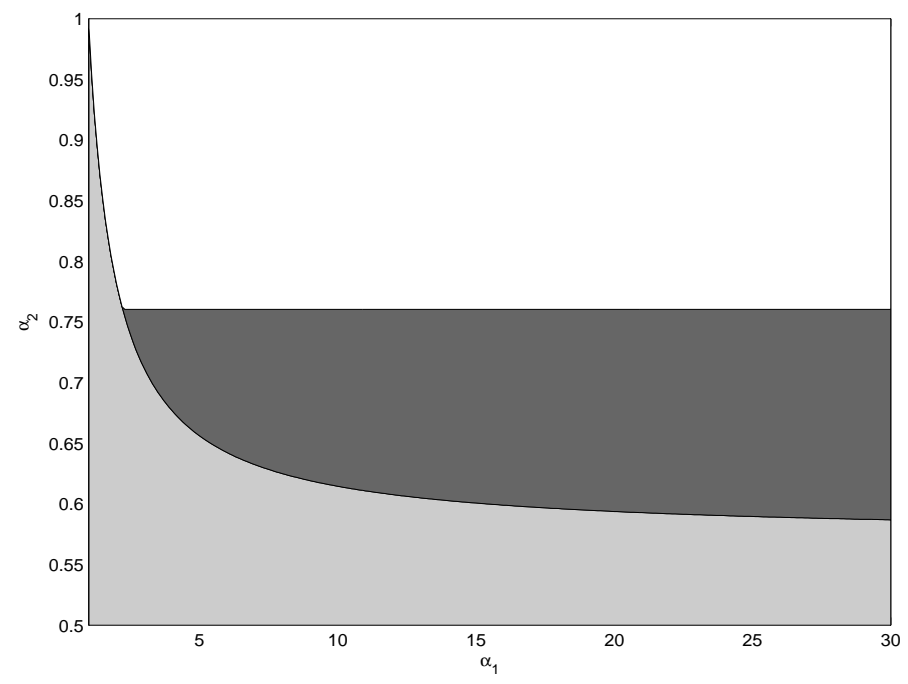

FigurE 3. Equilibrium characteristics: the new-Keynesian model with $\gamma_{1}=0.3, \gamma_{2}=0.1, \beta=0.99, \sigma=2.84, \kappa=0.3, p_{11}=0.8$, and $p_{22}=0.9$. The union of the dark and light shaded areas is an indeterminacy region according to Corollaries 1 and 2. The dark-shaded area represents the difference between Corollary 1 and Corollary 2 .

Figure 4 corresponds to the upper-right panel of Figure 2 in Davig and Leeper (2005, 2006b). It plots $\alpha_{1}$ against $\alpha_{2}$ when $\gamma_{1}=0, \gamma_{2}=0, \beta=0.99, \sigma=1.0$, 
$\kappa=0.17, p_{11}=0.8$, and $p_{22}=0.95$. If one uses Corollary 1 as though it were a necessary and sufficient condition, the dark-shaded area will be regarded as a uniqueness region and thus the inflation hawk in the active regime could respond to inflation strongly enough (by increasing the value of $\alpha_{1}$ ) to ensure that both regimes are determinate. This conclusion is incorrect since Corollary 2 establishes that there exist multiple bounded equilibria at every point in this dark-shaded area. If researchers were to use Corollary 1 to test for indeterminacy, using methods similar to those of Lubik and Schorfheide (2004), they would wrongly conclude that active monetary policy in the Volker-Greenspan era had ensured a unique equilibrium not only in the post-1982 period but also before 1980 .

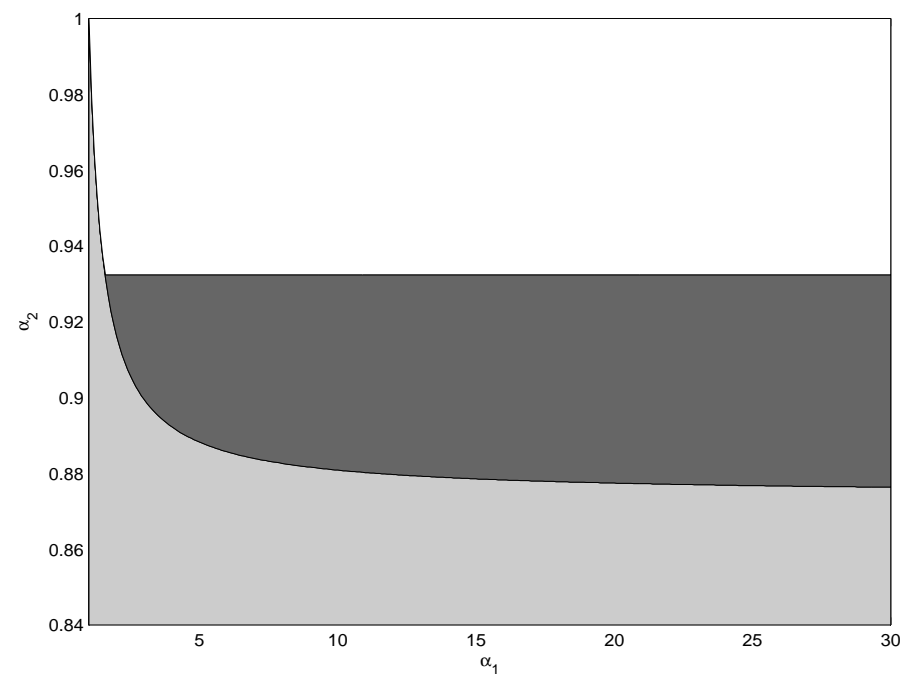

FiguRE 4. Equilibrium characteristics: the new-Keynesian model with $\gamma_{1}=0, \gamma_{2}=0, \beta=0.99, \sigma=1.0, \kappa=0.17, p_{11}=0.8$, and $p_{22}=0.95($ an example of DL's Figure 2). The union of the dark and light shaded areas is an indeterminacy region according to Corollaries 1 and 2. The darkshaded area represents the difference between Corollary 1 and Corollary 2 .

Corollaries 1 and 2 are straightforward to implement. Theorem 1 identifies an indeterminacy region that is larger than that identified by its two corollaries. To see whether this difference matters in practice, Figure 5 replicates the upper-right panel of Figure 2 from Davig and Leeper $(2005,2006 \mathrm{~b})$. This figures plots $\alpha_{1}$ against $\alpha_{2}$ when $\gamma_{1}=0, \gamma_{2}=0, \beta=0.99, \sigma=1.0, \kappa=0.17, p_{11}=0.95$, and $p_{22}=0.8$. The light-shaded area is identified by Corollary 1. For the most part, the area identified by Corollary 2 overlaps with the light-shaded area (and thus we do not plot it). The dark-shaded area marks the difference between Theorem 1 and its corollaries. 


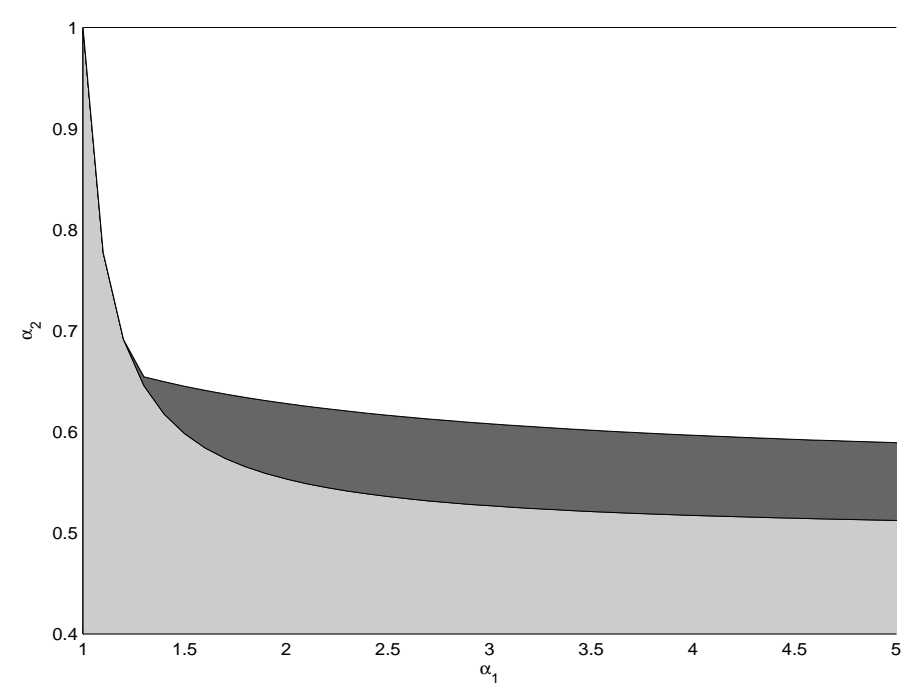

FigurE 5. Equilibrium characteristics: the new-Keynesian model with $\gamma_{1}=0, \gamma_{2}=0, \beta=0.99, \sigma=1.0, \kappa=0.17, p_{11}=0.95$, and $p_{22}=0.8$ (an example of DL's Figure 2). The union of the dark and light shaded areas is an indeterminacy region according to Theorem 1. The darkshaded area marks the difference between Theorem 1 and its corollaries.

\section{CONCLUSiOn}

The Taylor rule is widely regarded as an effective way to describe the historical conduct of monetary policy although the parameters of the rule have changed over time as documented by Clarida, Galí, and Gertler (2000), Lubik and Schorfheide (2004), and Boivin and Giannoni (2006). These changes are likely to be embedded in the public's perception that future monetary policy may change for better or worse (Goodfriend, 1993; Sargent, 1999; Mishkin, 2004). What are the equilibrium consequences if the public believes that there is a probability that monetary policy will at times abandon its hawkish stance on inflation in order to accommodate other economic concerns? This question is at the heart of our paper.

We have studied a version of the new-Keynesian model where parameters of the Taylor rule change over time according to a Markov-switching process. In this regimeswitching environment, rational agents form expectations by explicitly taking into account the probability of future policy changes between active and passive regimes. This environment is different from the basic new-Keynesian model with no regime switching and, since agents are forward looking, it differs substantially from backwardlooking Markov-switching models studied by Sims and Zha (2006). New approaches are required.

This paper has made four contributions to the study of NK models with endogenous regimes. First, we have provided a wide class of sufficient conditions for nonuniqueness of the bounded equilibrium. Second, we have proved that Davig and Leeper 
(2005, 2006b)'s condition for uniqueness is necessary but not sufficient and when used for identifying indeterminacy, is a special case of our general sufficient condition. Third, we have showed that a passive policy may spillover into an active regime and we have used existing estimates of policy for pre-1980 and post-1982 regimes to show that this phenomenon cannot be ruled out as descriptive of U.S. data. Finally, we have showed that there may exist regimes that are so passive that equilibria will be indeterminate in all regimes no matter what policy is followed by an active policy maker.

A challenging task for future research is to derive a necessary and sufficient condition under which there is a unique bounded equilibrium for the Markov switching new-Keynesian model. The techniques used to construct our examples are easy to implement and will, we hope, point the way to researchers who might wish to take up this challenge. Solving this problem is a necessary step if a researcher wished to follow Lubik and Schorfheide (2004) by constructing a test for indeterminacy in the U.S. economy that takes account of regime switches.

\section{Appendix A. Conditioning}

The results obtained in this paper rely heavily on the concept of conditioning in probability theory. Understanding this concept is essential to knowing where DL go wrong with their necessary and sufficient condition for determinacy in the NK model. The error occurs when they introduce the four new random variables $\pi_{1 t}, \pi_{2 t}, x_{1 t}$, and $x_{2 t}$ and use Eqs (5) and (6), reproduced below as Eqs (A1) and (A2),

$$
\begin{aligned}
& E_{t} \pi_{t+1}=p_{s_{t} 1} E_{t} \pi_{1 t+1}+p_{s_{t} 2} E_{t} \pi_{2 t+1}, \\
& E_{t} x_{t+1}=p_{s_{t} 1} E_{t} x_{1 t+1}+p_{s_{t} 2} E_{t} x_{2 t+1},
\end{aligned}
$$

to obtain the expanded linear system (7). The rules of conditioning, however, imply the following conditional relationships

$$
\begin{aligned}
& E_{t} \pi_{t+1}=p_{s_{t} 1} E_{t}\left[\pi_{t+1} \mid s_{t+1}=1\right]+p_{s_{t} 2} E_{t}\left[\pi_{t+2} \mid s_{t+1}=2\right], \\
& E_{t} x_{t+1}=p_{s_{t} 1} E_{t}\left[x_{t+1} \mid s_{t+1}=1\right]+p_{s_{t} 2} E_{t}\left[x_{t+2} \mid s_{t+1}=2\right] .
\end{aligned}
$$

Although $\pi_{i, t+1}=\pi_{t+1}$ and $x_{i, t+1}=x_{t+1}$ when $s_{t+1}=i$, it does not follow that $E_{t} \pi_{i, t+1}=E_{t}\left[\pi_{t+1} \mid s_{t+1}=i\right]$ and $E_{t} x_{i, t+1}=E_{t}\left[x_{t+1} \mid s_{t+1}=i\right]$ for $i=1,2$. The dynamics of $\pi_{i, t+1}$ and $x_{i, t+1}$ when $s_{t+1} \neq i$ affect the value of the $E_{t}[\cdot]$ operator. In order for (A1) and (A2) to hold, there must be additional hidden restrictions on the dynamics of $\pi_{i, t+1}$ and $x_{i, t+1}$ when $s_{t+1} \neq i$. The linear system represented by (7) is not the same as the original non-linear model (4). Although these two systems share some solutions in common, there may exist bounded solutions to the original model that are not solutions to the expanded linear system, as shown by the results in our paper. 


\section{Appendix B. Proof of Theorem 1}

Because any bounded solution $y_{t+1}$ of (8) can be written as

$$
y_{t+1}=\hat{y}_{t+1}+\tilde{y}_{t+1}
$$

where $\hat{y}_{t+1}$ is any particular bounded solution of $\mathrm{Eq}(8)$ and $\tilde{y}_{t+1}$ is a bounded solution of

$$
E_{t}\left[y_{t+1}\right]=\Gamma_{s_{t}} y_{t}
$$

Eq (8) has multiple bounded solutions if and only if Eq (A5) has a non-zero bounded solution, assuming a solution of $E q(8)$ exists. Since we are interested in the existence of multiple bounded solutions, we will operate under the assumption that there exists at least one bounded solution of $\mathrm{Eq}(8)$. This is a rather mild assumption. For instance, Eq (8) will have a solution of the form $y_{t}=G_{s_{t}} u_{t}$ if and only if there is $n h \times m$ matrix $G$ such that

$$
\operatorname{diag}\left(\Gamma_{i}\right) G=\left(P \otimes I_{n}\right) G \rho+\Psi
$$

where $\Psi^{\prime}=\left[\Psi_{1} \cdots \Psi_{h}\right]$.

Theorem 1 follows from the following more general theorem. While we believe that this theorem comes close to giving the full region on which there are multiple solutions, it is not practical to implement.

Theorem 2. Let $V_{1}, \cdots, V_{h}$ be linear subspaces of $\mathbb{C}^{n}$ with at least one of the $V_{i}$ non-zero and let $m_{1}, \cdots, m_{h}$ be positive real numbers. If there exist $n \times n$ complex matrices $\Lambda_{i, j}$ such that

and

$$
\begin{aligned}
\Lambda_{i, j} V_{j} & \subset V_{i}, \\
\left\|\Lambda_{i, j}\right\| & \leq \frac{m_{i}}{m_{j}},
\end{aligned}
$$

$$
\Gamma_{i} v_{i}=\sum_{j=1}^{h} p_{i, j} \Lambda_{j, i} v_{i} \text { for } v_{i} \in V_{i}
$$

then there exists multiple bounded solutions of Eq (A5).

Before proceeding with the proof of the theorem, we relate the conditions in this proposition to the constant parameter case. Consider the constant parameter analog of Eq (8),

$$
E_{t}\left[y_{t+1}\right]=\Gamma y_{t}-\Psi u_{t} .
$$

A bounded solution of Eq (A9) can be characterized by a linear subspace, often referred to as the stable matnifold, and a linear reduced form relation that describes the evolution of the solution. The linear subspaces $V_{i}$ play the role of the stable manifold and the matrices $\Lambda_{i, j}$ play the role of the reduced form coefficients. Equation (A6) ensures that the solutions stays on the stable manifold and Equation (A8) ensures 
that we indeed have a solution as long as we are on the stable manifold. Equation (A7) guarantees that the solution is bounded. One should note that Eq (A7) is stated in terms of a matrix norm, while the more usual conditions for the stable manifold are in terms of eigenvalues. While these conditions are related, they are not the same.

Proof. We inductively construct a non-zero bounded solution of Eq (A5). For $1 \leq$ $i \leq h$, choose $v_{i} \in V_{i}$ so that at least one of the $v_{i}$ is non-zero. Let $y_{1}=v_{s_{1}}$ and $y_{t+1}=\Lambda_{s_{t+1}, s_{t}} y_{t}$. Condition (A6) guarantees that $y_{t} \in V_{s_{t}}$. This, together with condition (A8), yields

$$
\begin{aligned}
E_{t}\left[y_{t+1}\right] & =\sum_{j=1}^{h} p_{s_{t}, j} E_{t}\left[y_{t+1} \mid s_{t+1}=j\right] \\
& =\sum_{j=1}^{h} p_{s_{t}, j} \Lambda_{j, s_{t}} y_{t} \\
& =\Gamma_{s_{t}} y_{t} .
\end{aligned}
$$

So $y_{t}$ is a solution of Eq (A5). This solution is bounded since, using condition (A7),

$$
\begin{aligned}
\left\|y_{t+1}\right\| & =\left\|\Lambda_{s_{t+1}, s_{t}} \Lambda_{s_{t}, s_{t-1}} \cdots \Lambda_{s_{2}, s_{1}} v_{s_{1}}\right\| \\
& \leq\left\|\Lambda_{s_{t+1}, s_{t}}\right\|\left\|\Lambda_{s_{t}, s_{t-1}}\right\| \cdots\left\|\Lambda_{s_{2}, s_{1}}\right\|\left\|v_{s_{1}}\right\| \\
& \leq \frac{m_{s_{t+1}}}{m_{s_{1}}}\left\|v_{s_{1}}\right\|
\end{aligned}
$$

While we have constructed a non-zero bounded solution, it could be a complex. However, in this case, both the real and imaginary components will be bounded solutions of $\mathrm{Eq}(\mathrm{A} 5)$ and at least one will be non-zero.

Theorem 1 is essentially Theorem 2 applied to the case in which each of the $V_{i}$ are one-dimensional. We now proceed with the proof of Theorem 1

Proof. Suppose that there exists $v$ and $c_{i}$ as in the Theorem 1 and let $v_{i}$ be the $i^{\text {th }}$ $n$-dimensional block of $v$. Define $V_{i}$ to be the subspace spanned by $v_{i}$ and define $\Lambda_{i, j}$ and $m_{i}$ by

$$
\Lambda_{i, j}=\left\{\begin{array}{cc}
\frac{c_{j}}{\left\|v_{j}\right\|^{2}} v_{i} v_{j}^{H} & v_{j} \neq 0 \\
0 & v_{j}=0
\end{array} \text { and } m_{i}=\left\{\begin{array}{cc}
\left\|v_{i}\right\| & v_{i} \neq 0 \\
1 & v_{i}=0
\end{array},\right.\right.
$$

where $v_{j}^{H}$ is the the conjugate transpose of $v_{j}$. By construction, $\Lambda_{i, j}$ satisfies condition (A6). Also,

$$
\left\|\Lambda_{i, j}\right\|=\left\{\begin{array}{cc}
\left|c_{j}\right| \frac{m_{i}}{m_{j}} & v_{j} \neq 0 \\
0 & v_{j}=0
\end{array}\right.
$$


In either case, $\left\|\Lambda_{i, j}\right\| \leq \frac{m_{i}}{m_{j}}$ so condition (A7) holds. Finally, note that Eq (9) implies

$$
\begin{aligned}
\Gamma_{i} v_{i} & =\left(e_{i}^{\prime} \otimes I_{n}\right) \operatorname{diag}\left(\Gamma_{i}\right) v \\
& =\left(e_{i}^{\prime} \otimes I_{n}\right) \operatorname{diag}\left(c_{j}\right)\left(P \otimes I_{n}\right) v \\
& =c_{i} \sum_{j=1}^{h} p_{i, j} v_{j} \\
& =\sum_{j=1}^{h} p_{i, j}\left(\frac{c_{i}}{\left\|v_{i}\right\|^{2}} v_{j} v_{i}^{H}\right) v_{i} \\
& =\sum_{j=1}^{h} p_{i, j} \Lambda_{j, i} v_{i},
\end{aligned}
$$

where $e_{i}$ is the $i^{\text {th }}$ column of the $h \times h$ identity matrix. So condition (A8) holds.

\section{Appendix C. Proof of Corollaries 1 And 2}

Proof. If $(\lambda, u)$ is a eigenvalue-eigenvector pair of $\operatorname{diag}\left(\Gamma_{i}^{-1}\right)\left(P \otimes I_{n}\right)$ with $|\lambda| \geq 1$, then

$$
\left(\lambda I_{n h}-\operatorname{diag}\left(\Gamma_{i}^{-1}\right)\left(P \otimes I_{n}\right)\right) u=0
$$

or

$$
\left(\operatorname{diag}\left(\Gamma_{i}\right)-\frac{1}{\lambda}\left(P \otimes I_{n}\right)\right) u=0 .
$$

Corollary 1 follows from Theorem 1 by taking $c_{i}=1 / \lambda$ and $v=u$.

Proof. Suppose $(\lambda, u)$ is a eigenvalue-eigenvector pair of $\Gamma_{i}$ with $|\lambda| \leq p_{i, i}$. Define

$$
c_{j}=\left\{\begin{array}{cc}
\frac{\lambda}{p_{i, i}} & j=i \text { and } p_{i, i}>0 \\
0 & \text { otherwise }
\end{array}, v_{i}=\left\{\begin{array}{cc}
u & j=i \\
0 & j \neq i
\end{array} \text { and } v=\left[\begin{array}{c}
v_{1} \\
\vdots \\
v_{h}
\end{array}\right]\right.\right.
$$

An easy calculation shows that

$$
\left(\operatorname{diag}\left(\Gamma_{i}\right)-\left(\operatorname{diag}\left(c_{i}\right) \otimes I_{n}\right)\left(P \otimes I_{n}\right)\right) v=0,
$$

and so the second corollary follows from Theorem 1 . 


\section{REFERENCES}

Andolfatto, D., And P. Gomme (2003): "Monetary Policy Regimes and Beliefs," International Economic Review, 44(1), 1-30.

Boivin, J., And M. Giannoni (2006): "Has Monetary Policy Become More Effective?," Review of Economics and Statistics, 88(3), 445-462.

Clarida, R., J. Galí, and M. Gertler (2000): "Monetary Policy Rules and Macroeconomic Stability: Evidence and Some Theory," Quarterly Journal of Economics, CXV, 147-180.

Cooley, T. F., S. F. LeRoy, and N. Raymon (1984): "Econometric policy evaluation: Note," The American Economic Review, 74, 467-470.

Davig, T., And E. M. Leeper (2005): "Generalizing the Taylor Principle," National Bureau of Economic Research Working Paper No. 11874, the September 2006 revised version.

- (2006a): "Fluctuating Macro Policies and the Fiscal Theory," in NBER Macroeconomic Annual 2006, ed. by D. Acemoglu, K. Rogoff, and M. Woodford. MIT Press, Cambridge, MA, forthcoming.

- (2006b): "Generalizing the Taylor Principle," The American Economic Review, the preview version on the AER website.

FArmer, R. E., D. F. WAggoner, And T. Zha (2006a): "Indeterminacy in a Forward Looking Regime Switching Model," NBER Working Paper 12540.

— (2006b): "Minimal State Variable Solutions to Markov-Switching Rational Expectations Models," Unpublished Manuscript.

Galí, J. (2006): "Comment on 'Fluctuating Macro Policies and the Fiscal Theory'," in NBER Macroeconomic Annual 2006, ed. by D. Acemoglu, K. Rogoff, and M. Woodford. MIT Press, Cambridge, MA, forthcoming.

Goodfriend, M. (1993): "Interest Rate Policy and the Inflation Scare Problem: 1979Ü1992," Federal Reserve Bank of Richmond Economic Quarterly, 79(1), 1-23. King, R. G. (2000): "The New IS-LM Model: Language, Logic, and Limits," Federal Reserve Bank of Richmond Economic Quarterly, 86/3, 45-103.

Leeper, E. M. (1991): "Equilibria under 'Active' and 'Passive' Monetary and Fiscal Policies," Journal of Monetary Economics, 27, 129-147.

Leeper, E. M., And T. ZhA (2003): "Modest Policy Interventions," Journal of Monetary Economics, 50(8), 1673-1700.

Lubik, T. A., And F. Schorfheide (2003): "Computing Sunspot Equilibria in Linear Rational Expectations Models," Journal of Economic Dynamics $\&$ Control, 28, 273-285.

(2004): "Testing for Indeterminacy: An Application to U.S. Monetary Policy," The American Economic Review, 94(1), 190-219. 
Mishkin, F. S. (2004): "Why the Federal Reserve Should Adopt Inflation Targeting," International Finance, 7(1), 117-127.

Sargent, T. J. (1999): The Conquest of American Inflation. Princeton University Press, Princeton, New Jersey.

Schorfheide, F. (2005): "Learning and Monetary Policy Shifts," Review of Economic Dynamics, 8(2), 392-419.

Sims, C. A. (1982): "Policy Analysis with Econometric Models," Brookings Papers on Economic Activity, 1, 107-164.

(2002): "Solving Linear Rational Expectations Models," Computational Economics, 20(1), 1-20.

Sims, C. A., And T. Zha (2006): "Were There Regime Switches in US Monetary Policy?," The American Economic Review, 96, 54-81.

Svensson, L. E., And N. Williams (2005): "Monetary Policy with Model Uncertainty: Distribution Forecast Targeting," Manuscript, Princeton University.

TAylor, J. B. (1993): "Discretion versus Policy Rules in Practice," vol. 39 of Carnegie-Rochester Conference Series on Public Policy, pp. 195-214. Amsterdam.

Woodford, M. (2003): Interest and Prices: Foundations of a Theory of Monetary Policy. Princeton University Press, Princeton, New Jersey.

UCla, Federal Reserve Bank of Atlanta, Federal Reserve Bank of Atlanta 